\title{
Viscosities of Binary Liquid Mixtures of Acetylene Tetrachloride with Benzene, Toluene, p-xylene, Acetone \& Cyclohexane at 303.15K
}

\author{
Meenu Agrawal and Atri Deo Tripathi
}

Chemistry Department, College of Engineering, Teerthankar Mahaveer University, Moradabad-244001, India

\begin{abstract}
Viscosities which are accurate to $\pm 0.01 \mathrm{mP}$, have been measured for binary liquid mixtures of acetylene tetrachloride $\left(\mathrm{CHCl}_{2} \mathrm{CHCl}_{2}\right.$, hereafter abbreviated simply as ATC) with benzene, toluene, $\mathrm{p}$-xylene, acetone and cyclohexane at $303.15 \pm 0.01 \mathrm{~K}$. The values of the quantity $\Delta \eta$, which refer to the deviations of the experimental values of the dynamic viscosities of the mixtures from the mole fraction mixture law values, have been found to be negative for the systems ATC-benzene, ATC-toluene, ATC-p-xylene and ATC-cyclohexane. For ATC-acetone, $\Delta \eta$ has been found to be negative at low mole fractions of ATC and positive at high mole fractions. Also the values of the parameter $d$ have been calculated from the equation $\ln \eta=x_{1} \ln \eta_{1}+x_{2} \ln \eta_{2}+x_{1} x_{2} d$, where $\eta_{1} \& \eta_{2}$ refer to the dynamic viscosities of the two pure liquids 1 and 2 whose mole fractions in the mixtures are $x_{1} \& x_{2}$ respectively. The values of $d$ indicate the existence of specific interaction of ATC with benzene, toluene, $p$-xylene and acetone. The viscosity data have been analysed in the light of absolute reaction rate and free volume theories of liquid viscosity.
\end{abstract}

Keywords: Acetylene tetrachloride, benzene, toluene, specific interactions, viscometer, hydrogen bonding.

\section{INTRODUCTION}

Binary systems of acetylene tetrachloride $\left(\mathrm{CHCl}_{2} \mathrm{CHCl}_{2}\right)(\mathrm{ATC})$ with benzene, toluene, $\mathrm{p}$-xylene, acetone and cyclohexane are of considerable interest from the viewpoint of the existence of specific interaction between the components in the liquid state. The specific interaction of $\mathrm{CHCl}_{2} \mathrm{CHCl}_{2}$ with aromatics and acetone can be visualized to be due to the presence of four $\mathrm{Cl}$ atoms and two $\mathrm{H}$ atoms in $\mathrm{CHCl}_{2} \mathrm{CHCl}_{2}$, on account of which it can act as a $\sigma-$ acceptor toward, and be involved in hydrogen-bond formation with aromatics and acetone. The aromatics in their interaction with $\mathrm{CHCl}_{2} \mathrm{CHCl}_{2}$ will act as $\pi$-donors, whereas acetone will act as an $\mathrm{n}$-donor. The system of cyclohexane with $\mathrm{CHCl}_{2} \mathrm{CHCl}_{2}$, in which case only the dispersion, dipolar and induction forces are believed to be present, is of interest as it will act as a reference system. Extensive studies concerning the properties of these systems have not been made in the literature. However, measurements of Dielectric Properties of mixtures of Acetylene Tetrachloride with toluene and acetone were carried out at $308.15 \mathrm{~K}$ [1]. The measurements of excess enthalpy of mixing have been also made for the binary mixtures of ATC with acetone [2\} and the results obtained have been discussed from the viewpoint of specific interaction between the components of various mixtures, it was thought worthwhile to get further information concerning the formation of adducts of ATC with aromatics and

*Address correspondence to this author at the Chemistry Department, College of Engineering, Teerthankar Mahaveer University, Moradabad-244001, India; Tel: +919458444596; E-mail: atrideo@rediffmail.com acetone from viscosity data. Hence, in the present programme measurements of viscosity have been made for binary mixtures of ATC with benzene, toluene, p-xylene, acetone and cyclohexane at 303.15 $\mathrm{K}$ and the results obtained are interpreted in this paper.

\section{EXPERIMENTAL}

Binary mixtures were prepared by a weighing method, using an analytical balance-ohaus-200. The uncertainty in mole fraction was $5 \times 10^{-4}$.

Materials: Benzene and toluene which were of $A R$ or GR grade quality were purified in a manner similar to that given elsewhere $[1,3]$. $p$-xylene (Pfizer)of reagent grade was subjected to treatments with concentrated sulfuric acid, with distilled water, with dilute sodium carbonate solution, and again with distilled water. It was further dried over anhydrous calcium chloride and then subjected to fractional crystallizations which were followed by fractional distillations. Acetone and cyclohexane were purified and their purity was checked as described earlier [4,5].

The kinematic viscometer described by Tuan and Fuoss [6], used for the present measurements of viscosities of the various pure liquids and their binary mixtures at $303.15 \mathrm{~K} \pm 0.01 \mathrm{~K}$ and by following the procedure described elsewhere [7].

\section{EXPERIMENTAL PROCEDURE}

The viscometer was properly mounted vertically on a wooden stand which was specially constructed for this purpose. The viscometer was first calibrated by 
noting the flow times for acetone and cyclohexane at $303.15 \mathrm{~K}$, and then calculating the constants $\mathrm{A}^{\prime}$ and $\mathrm{B}^{\prime}$ of the equation

$$
\eta / \rho=\mathrm{A}^{\prime} \mathrm{t}-\frac{B^{\prime}}{t}
$$

where $\eta / \rho$ is kinematic viscosity, $\eta$ refers to the dynamic viscosity and $\rho$ is the density. By using the dynamic viscosity in centipoises, $\rho$ in in $\mathrm{g} \mathrm{cm}^{-3}$ and time $t$ in seconds, the values of the constants $A^{\prime}$ and $B^{\prime}$ may be evaluated for the kinematic viscometer used in the present programme. The measurements of kinematic viscosities were first made for pure liquids and their binary mixtures studied in the present programme. Kinematic viscosities will be converted to dynamic viscosities $\eta$ by use of densities which, for pure liquids and their mixtures were measured by using a vibrating tube densimeter (model DMA, Anton -Paar 60/602) [8] at $303.15 \mathrm{~K}$.

The precision in the measured values of $\eta$ is in the order of $\pm 0.01 \mathrm{mp}$.

\section{RESULTS \& DISCUSSIONS}

The experimental values of $\eta$ for the various pure liquids and their binary mixtures of ATC at $303.15 \mathrm{~K}$ are given in Table 1 and plotted in Figure 1 , where $x_{1}$ refers to the mole fraction of ATC. The values of $\eta$ for benzene, toluene, p-xylene, acetone, and ATC have been found to be $5.62,5.20,5.65,2.95$, and $14.62 \mathrm{mP}$ respectively at $303.15 \mathrm{~K}$, which are in good agreement with the corresponding literature values $5.66,5.23,5.68$, 2.954 , and $14.56 \mathrm{mP}$ [9] for the various liquids in the same order.

A simple additive relation [10] to predict the mixture viscosity from the properties of pure components, when the interactions of pure components are neglected, is the equation

$\ln \eta=x_{1} \ln \eta_{1}+x_{2} \ln \eta_{2}$

Where $\eta_{1}$ and $\eta_{2}$ refer to the dynamic viscosities of the two pure components 1 and 2 whose mole fractions are $x_{1} \& x_{2}$ respectively, and $\eta$ is the dynamic viscosity of the mixture. In practice, however, deviations of the experimental values of the dynamic viscosities of the mixtures from those predicted by Eq. (1) are found to occur, thus indicating that explicit account of the contributions to the mixture viscosity due to interactions between components has to be taken. We shall now analyze our viscosity data for the various mixtures in the light of the various theories of liquid mixture viscosity. The various theories of liquid mixtures have been discussed by Reid, Prausnitz \& Sherwood [11]. Two major semi empirical theories which can be used to predict liquid mixture viscosity are the absolute reaction rate theory of Eyring and coworkers [12] and the free volume theory [13-15]. The absolute reaction rate theory relates the viscosity to the free energy required by a molecule to overcome the attractive force field of its neighbours, so that it can jump to a new equilibrium position; and hence the deviation of the experimental value of the mixture viscosity from the value obtained from Eq (1) is related to the free energy of mixing or the excess free energy. On the other hand, the free volume theory relates the viscosity to the probability of occurrence of an empty neighbouring site into which a molecule can jump. This probability is exponentially related to the free volume of the liquid. Hence, the deviation of the mixture viscosity from Eq. (1) can be attributed to the variations in the free volume of the solution. Combining the absolute reaction rate and free volume theories of viscosity of liquids, Bloomfield and Dewan [10] have obtained the relation

$$
\begin{aligned}
& \ln \eta=x_{1} \ln \eta_{1}+x_{2} \ln \eta_{2}=\frac{\Delta H^{M}}{R T}+\frac{\Delta S^{R}}{R T}+\left[\frac{1}{\bar{v}-1}-\frac{x_{1}}{\bar{v}_{1}-1}-\frac{x_{2}}{\bar{v}_{2}-1}\right] \\
& =\ln \eta_{i d}+\ln \eta_{H}+\ln \eta_{s}+\ln \eta_{v}
\end{aligned}
$$

In Eq (2), $\Delta H^{M}$ is the enthalpy of mixing per mole of the solution, $\Delta S^{R}$ the residual entropy per mole, $\mathrm{R}$ the gas constant, $T$ the absolute temperature, and, $\tilde{U}_{1}, \tilde{U}_{2}$ and $\tilde{U}$ the reduced volumes of component 1 , component 2 and the solution respectively. In Eq. (2), $\eta_{\text {id }}$ refers to the contribution to the mixture viscosity from the ideal mixture viscosity obtained from Eq. (1), and $\eta_{H}, \eta_{s}$ and $\eta_{v}$ refer to the contributions to the liquid mixture viscosity from enthalpic, entropic and free volume, corrections respectively, to the ideal behavior. For estimating the contribution to the mixture viscosity from $\Delta H^{M} /(\mathrm{RT})$ and $\Delta S^{R} / \mathrm{R}$ in Eq. (2), $\Delta H^{M}$ and $\Delta S^{R}$ were obtained from Flory's equation [16] which can be written as

$$
\frac{\Delta H^{M}}{R T}=\frac{x_{1} C_{1}}{T_{1}}\left[\frac{1}{\bar{v}_{1}}-\frac{1}{\bar{v}}\right]+\frac{x_{2} C_{2}}{T_{2}}\left[\frac{1}{\bar{v}_{2}}-\frac{1}{\bar{v}}\right]+\frac{x_{1} C_{1} \theta_{2} x_{12}}{\bar{v} T_{i} * P_{i} *}
$$

And

$\frac{\Delta S^{R}}{R T}=-3 x_{1} C_{1} \ln \frac{\left(\bar{v}_{1}\right)^{1 / 3}-1}{(\bar{v})^{1 / 3}-1}-3 x_{2} C_{2} \ln \frac{\left(\bar{v}_{2}\right)^{1 / 3}-1}{(\bar{v})^{1 / 3}-1}$

In Eqs (3) and (4), the parameter $\mathrm{Ci}$ for a component $\mathrm{i}$ is related to the characteristics pressure $P^{i * *}$ the characteristic temperature $T^{*} i^{\prime}$ and the hard - 
Table 1: Values of Experimental and Calculated Viscosities (in cP), $\Delta \eta$ and Parameter $d$ for the Mixtures of Acetylene Tetrachloride with Benzene, Toluene, p-xylene, Acetone and Cyclohexane at 303.15K

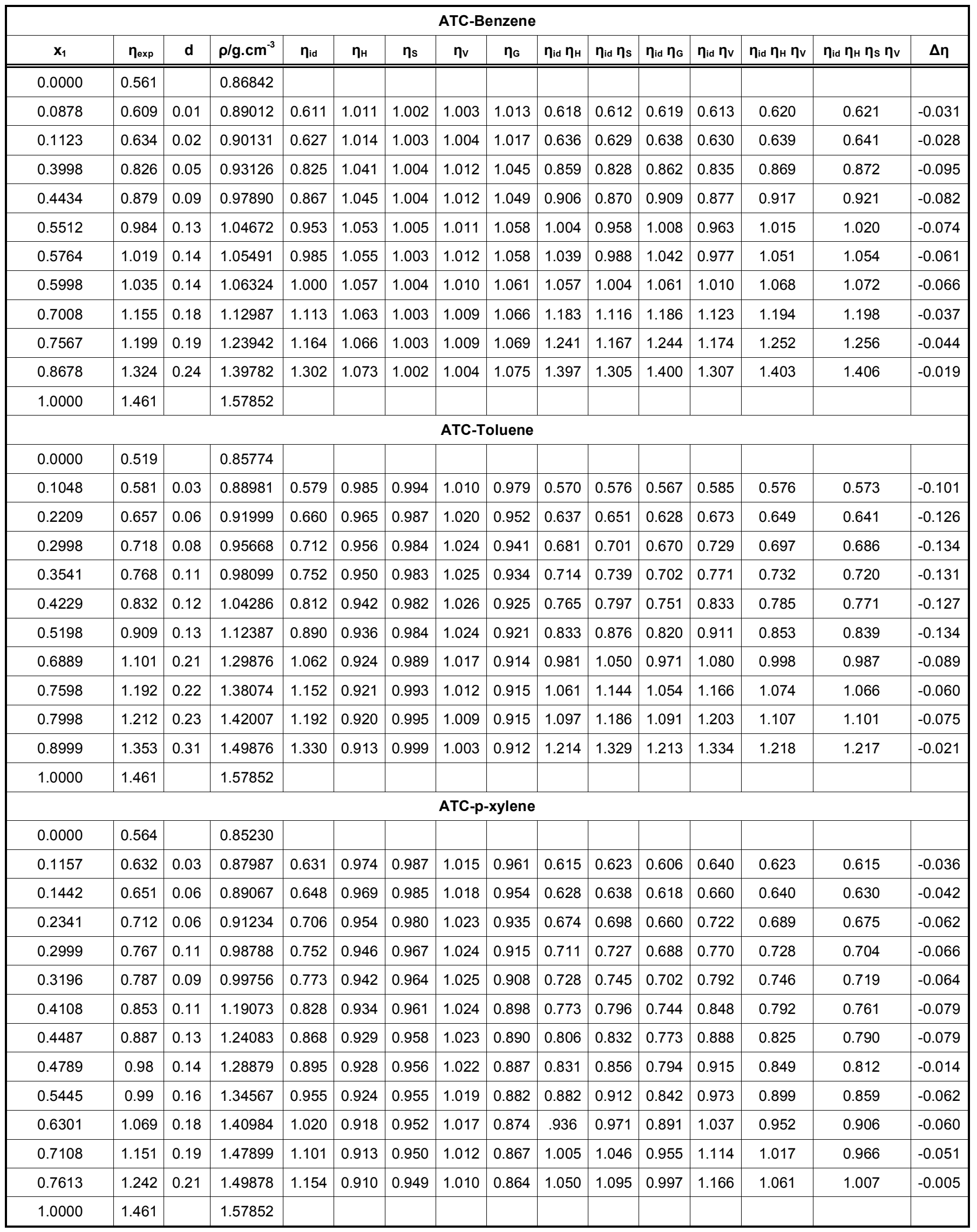


(Table 1). Continued.

\begin{tabular}{|c|c|c|c|c|c|c|c|c|c|c|c|c|c|c|c|}
\hline \multicolumn{16}{|c|}{ ATC-cyclohexane } \\
\hline 0.1645 & 0.875 & -0.26 & 0.79872 & 0.896 & 1.170 & 0.949 & 1.057 & 1.237 & 1.048 & 0.947 & 1.108 & 0.850 & 0.995 & 1.052 & -0.051 \\
\hline 0.1884 & 0.879 & -0.28 & 0.82010 & 0.907 & 1.142 & 0.988 & 1.018 & 1.163 & 1.036 & 0.923 & 1.055 & 0.896 & 1.023 & 1.041 & -0.062 \\
\hline 0.4036 & 0.971 & -0.27 & 0.98834 & 1.035 & 1.436 & 0.913 & 1.101 & 1.581 & 1.486 & 1.140 & 1.636 & 0.945 & 1.357 & 1.494 & -0.108 \\
\hline 0.5125 & 1.031 & -0.26 & 1.12209 & 1.096 & 1.540 & 0.910 & 1.105 & 1.702 & 1.688 & 1.211 & 1.865 & 0.997 & 1.535 & 1.696 & -0.118 \\
\hline 0.6613 & 1.153 & -0.21 & 1.32444 & 1.201 & 1.689 & 0.918 & 1.095 & 1.849 & 2.028 & 1.315 & 2.221 & 1.103 & 1.863 & 2.040 & -0.092 \\
\hline 0.7578 & 1.234 & -0.2 & 1.42672 & 1.260 & 1.763 & 0.929 & 1.081 & 1.906 & 2.221 & 1.362 & 2.402 & 1.171 & 2.064 & 2.231 & -0.073 \\
\hline 0.8862 & 1.298 & -0.19 & 1.48002 & 1.323 & 1.856 & 0.935 & 1.069 & 1.958 & 2.405 & 1.414 & 2.613 & 1.245 & 2.147 & 2.458 & -0.091 \\
\hline 1.0000 & 1.461 & & 1.57852 & & & & & & & & & & & & \\
\hline 0.1198 & 0.418 & 1.26 & 0.89993 & 0.357 & 0.950 & 0.968 & 1.069 & 0.920 & 0.339 & 0.346 & 0.328 & 0.382 & 0.363 & 0.351 & -0.016 \\
\hline 0.1554 & 0.445 & 1.25 & 0.94463 & 0.377 & 0.938 & 0.961 & 1.084 & 0.901 & 0.354 & 0.362 & 0.340 & 0.409 & 0.384 & 0.369 & -0.030 \\
\hline 0.198 & 0.489 & 1.23 & 0.98767 & 0.396 & 0.927 & 0.956 & 1.097 & 0.886 & 0.367 & 0.379 & 0.351 & 0.434 & 0.402 & 0.384 & -0.036 \\
\hline 0.2343 & 0.562 & 1.2 & 1.09890 & 0.421 & 0.915 & 0.950 & 1.111 & 0.869 & 0.385 & 0.400 & 0.366 & 0.468 & 0.428 & 0.407 & -0.005 \\
\hline 0.3102 & 0.634 & 1.21 & 1.14532 & 0.480 & 0.889 & 0.940 & 1.135 & 0.836 & 0.427 & 0.451 & 0.401 & 0.545 & 0.485 & 0.456 & -0.022 \\
\hline 0.3312 & 0.651 & 1.21 & 1.16122 & 0.497 & 0.883 & 0.938 & 1.139 & 0.828 & 0.439 & 0.466 & 0.412 & 0.566 & 0.500 & 0.469 & -0.030 \\
\hline 0.3545 & 0.689 & 1.2 & 1.18909 & 0.520 & 0.875 & 0.935 & 1.145 & 0.818 & 0.455 & 0.486 & 0.425 & 0.595 & 0.521 & 0.487 & -0.019 \\
\hline 0.4576 & 0.845 & 1.33 & 1.20786 & 0.604 & 0.849 & 0.929 & 1.157 & 0.789 & 0.513 & 0.561 & 0.477 & 0.699 & 0.593 & 0.551 & 0.017 \\
\hline 0.9452 & 1.452 & 1.13 & 1.55789 & 1.318 & 0.772 & 0.960 & 1.044 & 0.741 & 1.017 & 1.265 & 0.977 & 1.376 & 1.062 & 1.020 & 0.055 \\
\hline 1.0000 & 1.461 & & 1.57852 & & & & & & & & & & & & \\
\hline
\end{tabular}

core volume per mole $V i^{*}$ of component i as described elsewhere $[7,15]$. The characteristics parameters $P i^{\prime *}$ $T i^{* \prime}$ and $V i^{* *}$ and the reduced volume ũi which are given in Table 2, and the reduced temperature Ti of the pure component $\mathrm{i}$ used in the present calculation were obtained from the values (see Table 2) of the molal volume $V$, the thermal expansion coefficient $\alpha$, and the thermal expansion coefficient $\gamma$, by using the methods described by Abe and Flory [16]. The parameter $\theta_{2} \mathrm{X}_{12}$ (characteristic of a system), used to calculate $\Delta H^{M}$, (RT) from Eq. (3), was estimated from the reduced excess volume $\tilde{u}^{E}$, by using the experimental values of the excess volumes for equimolal mixtures and by employing the relations described by Abe and Flory [16]. The values of the reduced volumes (Ũ) of the solutions used in the present calculations were also obtained from the relations of Abe and Flory [16] by using the experimental values of excess volumes for the various systems [5].

The contributions of the various terms in Eq. (2) are given in columns 3-6 of Table 1, and values of the free energy contribution, defined by $\eta_{G}=\eta_{H} \eta_{S}$ are given in column 9 . The various combinations of the calculated contributions from various terms to $\eta$, combining them multiplicatively, in accordance with the logarithmic relation in Eq. (2), are recorded in columns 10-13 of Table 1. The free volume theory, which takes into account free volume corrections to the ideal mixture viscosity $\eta_{\text {id }}$, corresponds to $\eta_{\text {id }} \eta_{v}$, where as absolute 


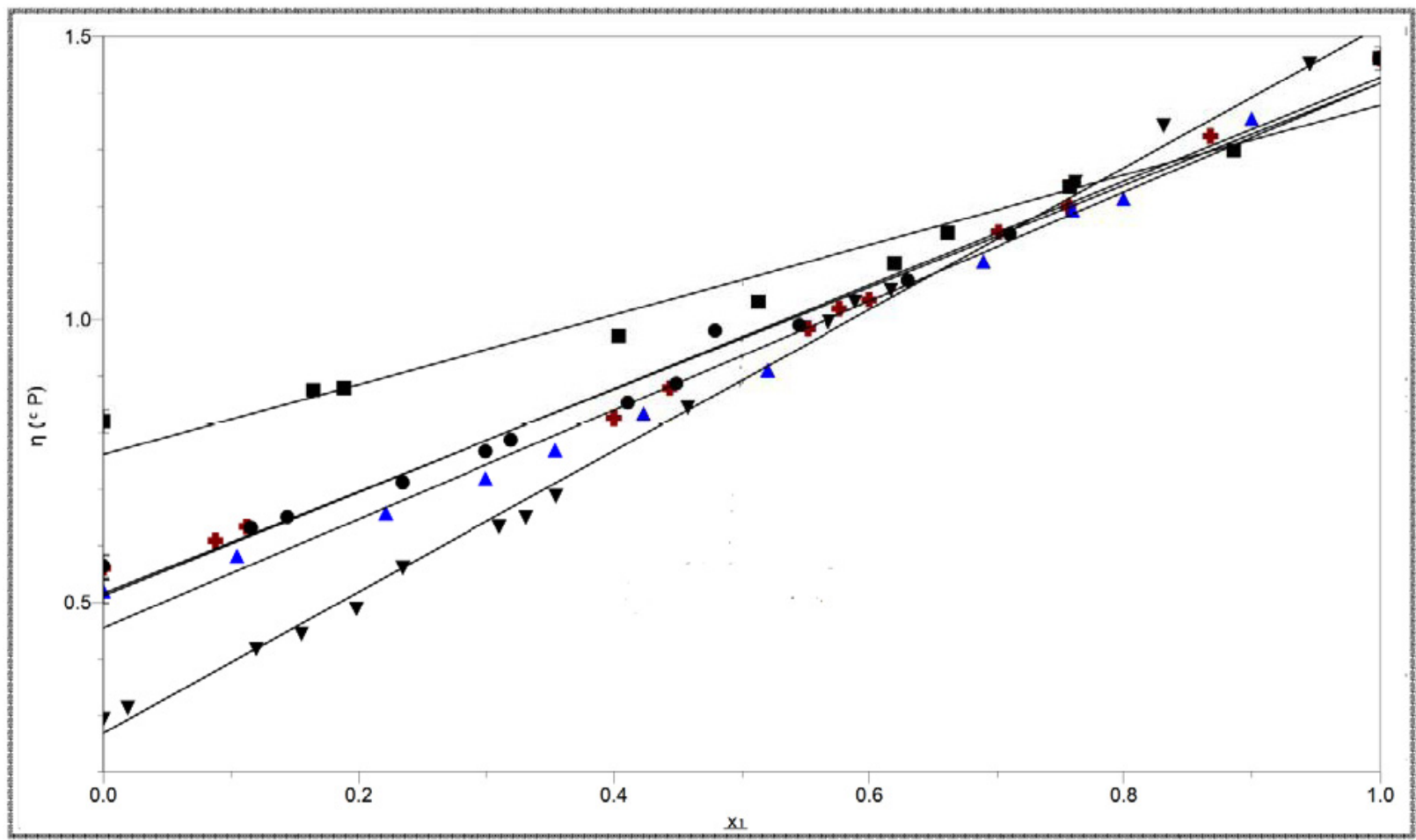

Figure 1: Plot of dynamic Viscosity Vs mole fraction $x 1$ of Acetylene Tetrachloride + benzene,++ toluene $\Delta,+p-x y l e n e ~$ +cyclohexane $\mathbf{\square},+$ acetone $\boldsymbol{\nabla}$ at $303.15 \mathrm{~K}$.

Table 2: Parameters for Pure Liquids at $303.15 \mathrm{~K}$

\begin{tabular}{|c|c|c|c|c|c|c|c|}
\hline Liquid & $\begin{array}{c}V \\
\mathrm{~cm}^{3} \mathrm{~mol}^{-1}\end{array}$ & $\begin{array}{l}\alpha \times 10^{3} \\
\operatorname{deg}^{-1}\end{array}$ & $\frac{\mathrm{Y}}{\text { cal } \mathrm{cm}^{-3} \mathrm{deg}^{-1}}$ & ũ & $\begin{array}{c}\mathrm{V}^{*} \\
\mathrm{~cm}^{3} \mathrm{~mol}^{-1}\end{array}$ & $\begin{array}{l}\mathrm{T}^{*} \\
\mathrm{~K}\end{array}$ & $\begin{array}{c}\mathrm{P}^{*} \\
\mathrm{cal} \mathrm{cm}^{-3}\end{array}$ \\
\hline Benzene & $89.95^{a}$ & $1.233^{\mathrm{b}}$ & $0.292^{b}$ & 1.2975 & 69.33 & 4730 & 149 \\
\hline Toluene & $107.43^{a}$ & $1.089^{c}$ & $0.278^{d}$ & 1.2693 & 84.64 & 5036 & 136 \\
\hline p-xylene & $124.57^{\mathrm{a}}$ & $1.038^{\mathrm{C}}$ & $0.268^{e}$ & 1.2590 & 98.64 & 5165 & 129 \\
\hline Cyclohexane & $109.40^{a}$ & $1.232^{c}$ & $0.247^{b}$ & 1.2973 & 84.33 & 4732 & 126 \\
\hline Acetone & $74.53^{a}$ & $1.477^{\circ}$ & $0.258^{d}$ & 1.3422 & 55.53 & 4354 & 141 \\
\hline ATC & $106.33^{a}$ & $1.014^{c}$ & $0.340^{d}$ & 1.2540 & 84.79 & 5231 & 162 \\
\hline
\end{tabular}

${ }^{a}$ Based on densities reported in Table 1.

balues obtained from the interpolation of the data tabulated in Ref. [16]

'Values computed from densities at three temperatures, the density data being taken from Ref. [9].

${ }^{d}$ Values estimated from isothermal compressibility $k_{T}$, by using the relation $\gamma=\alpha / k_{T}$. Values of $k_{T}$ were obtained from Ref. [20].

'Vales estimated from isothermal compressibility which,in turn, was obtained from adiabatic compressibility reported in Ref. [9].

reaction rate theory, which takes into account the free energy corrections to the ideal mixture viscosity, corresponds to $\eta_{\text {id }} \eta_{G}$. Macedo - litovitz 's theory [17] which takes into account enthaplic and free volume corrections to ideal mixture viscosity corresponds to $\eta_{\text {id }}$ $\eta_{H} \eta_{V}$ which is given in column 14 of Table 1 . The values of the complete product $\eta_{i d} \eta_{H} \eta_{S} \eta_{V}$ are given in the column 15.

Table 1 shows that for the system ATC- benzene, the experimental viscosities are best reproduced by $\eta_{\text {id }}$ $\eta_{S}$ or $\eta_{\text {id }} \eta_{V}$, whereas the values predicted by $\eta_{\text {id }} \eta_{H}$ or $\eta_{\text {id }}$ $\eta_{v}$ are somewhat less satisfactory in this case. Further, the experimental viscosities for the systems ATCtoluene and ATC - $p$ - xylene are best reproduced by $\eta_{\text {id }}$ $\eta_{\mathrm{V}}$. For the system ATC- cyclohexane, the experimental viscosities are also best reproduced by $\eta_{\text {id }} \eta_{\mathrm{V}}$, whereas the values predicted by $\eta_{\text {id }} \eta_{H}, \eta_{\text {id }} \eta_{S}, \eta_{\text {id }} \eta_{G}, \eta_{\text {id }} \eta_{H} \eta_{V}$ and $\eta_{i d} \eta_{H} \eta_{S} \eta_{V}$ exhibit larger deviations from the experimental values $\eta$ for this system. Though the values predicted by, $\eta_{\text {id }} \eta_{V}$ for ATC -ACETONE are relatively closer to the experimental values, none of $\eta_{\text {id }}$ $\eta_{H}, \eta_{\text {id }} \eta_{S}, \eta_{\text {id }} \eta_{G}, \eta_{\text {id }} \eta_{V}, \eta_{\text {id }} \eta_{H} \eta_{V}$ and $\eta_{\text {id }} \eta_{H} \eta_{S} \eta_{V}$ satisfactorily reproduces the experimental viscosities for this system . 
The experimental values of $\eta$ for the various mixtures have been used to calculate the values of the quantity $\Delta \eta$ from the relation

$\Delta \eta=\eta-x_{1} \eta_{1}-x_{2} \eta_{2}$

Where $\eta_{1}, \eta_{2}, \eta, x_{1} \& x_{2}$ have the same significance as in eq. (1). The values of $\Delta \eta$ for the various system of ATC have been given in the last column of Table 1, which shows that the values of $\Delta \eta$ are negative throughout the whole range of composition for ATCbenzene, ATC-toluene, ATC-p- xylene, and ATCcyclohexane. It also shows that the values of $\Delta \eta$ are negative throughout the whole range of composition for ATC-benzene, ATC-toluene, ATC-p-xylene, and ATCcyclohexane.

It also shows that for ATC-acetone, the values of $\Delta \eta$ are negative at low mole fractions of ATC and positive at high mole fractions. At $x_{1}=0.5$, the values of $\Delta \eta$ have the sequence

$(\Delta \eta)_{\text {acetone }}>(\Delta \eta)_{\text {p-xylene }}>\left((\Delta \eta)_{\text {benzene }}>(\Delta \eta)_{\text {toluene }}>>\right.$ $(\Delta \eta)_{\text {cyclohexane }}$

The values of $\Delta \eta$ can be discussed from the viewpoint of the existence of specific interaction leading to the formation of molecular complexes between the components of the various systems [7]. For systems where dispersion, induction and dipolar forces are operating, the values of $(\Delta \eta)$ are found to be negative, whereas the existence of specific interaction leading to the formation of adducts between the two components of various binary systems tends to make $\Delta \eta$ positive. The measurements of dielectric constants [1] have indicated that ATC forms molecular complexes with the aromatic hydrocarbons and acetone. The large negative values of $\Delta \eta$ for ATC-benzene, ATC-toluene and, ATC-p-xylene can be attributed to the predominance of contributions to $\Delta \eta$ on account of the dispersion, dipolar and induction forces over those due to specific interactions between the components. The slightly negative values of $\Delta \eta$ at low mole fractions and positive values of $\Delta \eta$ at high mole fractions of ATCacetone system show that acetone forms molecular complexes with ATC. From an NMR study McClellan and Nicksic [18] have shown that the molecules of ATC are self associated through hydrogen bonding. The specific interaction are expected to be hydrogen bond formation between the hydrogen of ATC and proton acceptor groups.
According to Grunberg and Nissan, [19], the values of $\eta$ for a binary mixture of components 1 and 2 is given by

$\ln \eta=x_{1} \ln \eta_{1}+x_{2} \ln \eta_{2}+x_{1} x_{2} d$

Where $d$ is a parameter which is regarded as a measure of the strength of interaction between the components. The values of $d$, as estimated from eq (3) by taking $\eta_{1}, \eta_{2}, \eta$ in $\mathrm{mP}$ are given in the column 3 of the Table 1 for various mixtures. The values of $d$ which are slightly positive in the case of the systems ATCbenzene, ATC-toluene, ATC-p-xylene, suggest that ATC forms weak complexes with benzene, toluene and $p$-xylene; whereas the higher positive values of $d$ for ATC -acetone system show that ATC forms strong complexes with acetone in the liquid state because of hydrogen bonding between the hydrogen atom of ATC and lone pair of electrons on the oxygen atom of acetone. On the other hand, the specific interaction between ATC and aromatic hydrocarbons can be explained as due to formation of a weak hydrogen bond between the hydrogen atom of ATC and $\pi$ electrons of the aromatic ring. However, there is also a possibility of charge transfer complex formation with aromatic hydrocarbons via chlorine atom- $\pi$-electron interactions.

\section{ACKNOWLEDGEMENT}

Authors are extremely grateful to Professor N.K. Joshi, Principal, College of Engineering, Teerthankar Mahaveer University, Moradabad, UP (India)-244001 for encouragement during course of these investigations.

\section{REFERENCES}

[1] Tripathi AD. Dielectric Properties of Mixtures of Acetylene Tetrachloride with Toluene and Acetone. IJ Solution Chem 1994; 23(7): 769-76.

[2] Pathak G, Tripathi AD, Pradhan S. The excess molar enthalpies of mixing of 1, 1,2, 2-tetrachloroethane with acetone, n-dibutyl ether, acetonitrile and dimethylsulphoxide. Thermochim Acta 1992; 197: 329-33. http://dx.doi.org/10.1016/0040-6031(92)85031-P

[3] Tripathi AD. Excess molar enthalpies of Dibromomethane with benzene, methanol, dimethylsulfoxide and pyrrolidin- 2one at 303.15 K. J Chem Eng Data 2010; 55: 1421-23. http://dx.doi.org/10.1021/je9005882

[4] Tripathi AD. Excess enthalpies of Dibromomethane with acetone, 1, 4-dioxane, pyridine, diethyl ether, ethyl methyl ketone and tetrahydrofuran at $303.15 \mathrm{~K}$. J Chem Eng Data 2010; 55: 1113-16. http://dx.doi.org/10.1021/je900547w

[5] Nath J, Tripathi AD. Binary systems of 1,1,2,2 tetrachloroethane with benzene, toluene, p-xylene, acetone, and cyclohexane. 1. Excess volumes, ultrasonic velocities, 
and adiabatic compressibilities at 298.15 and $308.15 \mathrm{~K}$. J Chem Eng Data 1983; 28: 263-66.

http://dx.doi.org/10.1021/je00032a038

[6] Tuan DFT, Fuoss RM. Electrostriction in polar solvents. J Phys Chem 1963; 67(6): 1343-47.

http://dx.doi.org/10.1021/j100800a043

[7] Nath J, Narain B. Binary systems of tetrachloroethylene with benzene, toluene, p-xylene, carbon tetrachloride and cyclohexane. 2 Viscosities at 303.15K. J Chem Eng Data 1983; 28: 296-97. http://dx.doi.org/10.1021/je00033a003

[8] Tripathi AD. Heat capacities of tetra phenyl phosphonium chloride in methanol; ethanol; acetonitrile and water Fluid Phase Equilibria 2010; 293: 205-208. http://dx.doi.org/10.1016/j.fluid.2010.03.017

[9] TImmermans J. Physlco-Chemlcal Constants of Pure Organic Compounds. Elsevier: Amsterdam 1950.

[10] Bloomfield VA, Dewan RK. Viscosity of liquid mixtures. J Phy Chem (USA) 1971; 75: 3113-19. http://dx.doi.org/10.1021/j100689a014

[11] Reid RC, Prausnitz JM, Sherwood TK. McGraw-Hill, $3^{\text {rd }}$ ed. The Properties of Gases \& Liquids.

[12] Glass Tone S, Laidler KJ, Eyring $\mathrm{H}$. The theory of rate processes (McGraw Hill, New York) 1941; Ch. 9.

[13] Doolittle AK. J Appl Phys (USA) 1951; 22: 1471; 23(1952) 236.
[14]

Williams ML, Landel RF, Ferry JD. Mechanical Properties of Substances of High Molecular Weight .19. The Temperature Dependence of Relaxation Mechanisms in Amorphous Polymers and Other Glass-Forming Liquids. J Am Chem Soc 1955; 77. J Am Chem Soc (USA) 1955; 77: 3701-707. http://dx.doi.org/10.1021/ja01619a008

[15] Cohen $\mathrm{MH}$, Turnbull D. Molecular transport in liquids and glasses. J Chem Phy (USA) 1959; 31: 1164-69. http://dx.doi.org/10.1063/1.1730566

[16] Flory PJ. Statistical Thermodynamics of Liquid Mixtures. J Am Chem Soc (USA) 1965; 87: 1833-38. DOI: 10.1021/ja01087a002

[17] Macedo PB, Litovitz TA. On the Relative Roles of Free Volume and Activation Energy in the Viscosity of Liquids. J Chem Phy (USA) 1965; 42: 245. http://dx.doi.org/10.1063/1.1695683

[18] McClellan AL, Nick sic SW. Simultaneous, Independent Hydrogen-Bonding Equilibria and Self-Association in Some Halomethanes and Haloethanes. J Phy Chem 1965; 69: 44649.

http://dx.doi.org/10.1021/j100886a014

[19] Grunberg L, Nissan AH. Mixture Law for Viscosity Nature 1949; 164: 799-800. http://dx.doi.org/10.1038/164799b0

[20] Weast RC. Handbook of Chemistry and Physics (The Chemical Rubber Co Cleveland, Ohio, USA) 1971. 